\title{
Medical Image of the Week: Pulmonary Arteriovenous Fistula
}
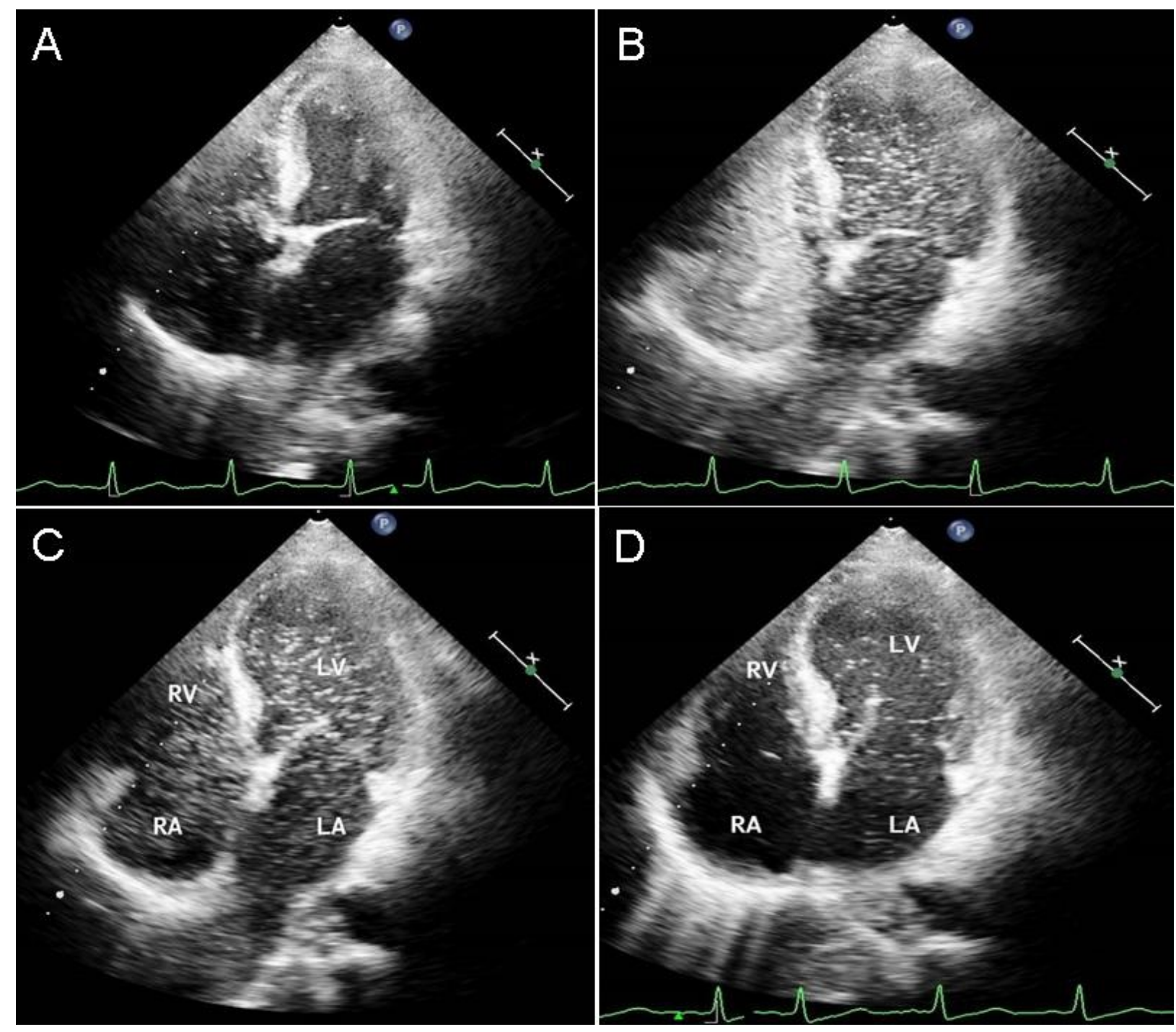

Figure 1. Panel A: Micro-bubbles appear in the right atrium (RA) and right ventricle (RV) with delayed appearance in the left atrium (LA) and left ventricle (LV). Panels B and C: The density of the micro-bubbles were same in the left and the right cardiac chambers even after 10 cardiac cycles. Panel D: When the injection was stopped, there were micro-bubbles in the left cardiac chambers, but none in the right cardiac chambers.

A 60 year-old man with hepatic cirrhosis, was referred for chest pain, shortness of breath, and progressive cyanosis and an echocardiographic evaluation. $\mathrm{PaO} 2$ was $64 \mathrm{~mm} \mathrm{Hg}$ on room air, but only $74 \mathrm{~mm} \mathrm{Hg}$ on $100 \%$ oxygen. Chest X-ray and pulmonary function testing were normal. A contrast echocardiography using agitated saline (bubble study) was performed. A delayed appearance of a substantial amount of micro-bubbles in the left atrium greater than three 
cardiac cycles after appearance in the right atrium and ventricle was suggestive of pulmonary arteriovenous fistula (Figure 1A). The delayed appearance and a large amount of micro-bubbles in the left atrium preclude the intracardiac shunting result of a patent foramen ovale (PFO) or atrial septal defect (ASD). Interestingly, the density of micro-bubbles were same in the left and the right cardiac chambers even after 10 cardiac cycles (Figure 1B and 1C). When the injection was stopped, there were micro-bubbles in the left cardiac chambers, but none in the right cardiac chambers (Figure 1D). Although pulmonary angiography remains the gold standard method for definitive diagnosis of the pulmonary arteriovenous malformations, contrast echocardiography can suggest arteriovenous fistula in the setting of unexplained hypoxemia before angiography, especially in hospitals without on-site angiography facilities.

Manisha Bajracharya MD, Madhu Gupta MD, Liping Chen MD PhD

Department of Gynecology and the Cardiovascular Disease Center, Norman Bethune College of Medicine, Jilin University, Changchun, China

\section{Reference}

Nanthakumar K, Graham AT, Robinson TI, Grande P, Pugash RA, Clarke JA, Hutchison SJ, Mandzia JL, Hyland RH, Faughnan ME. Contrast echocardiography for detection of pulmonary arteriovenous malformations. Am Heart J. 2001;141(2):243-6. [CrossRef] [PubMed] 